\title{
GPS Position Estimation Using Integer Ambiguity Free Carrier Phase Measurements
}

\author{
G Sateesh Kumar ${ }^{\# 1}$, M N V S S Kumar ${ }^{\# 2}$, G Sasi Bhushana Rao *3 \\ \# Dept. of ECE, Aditya Institute of Technology and Management Tekkali, Andhra Pradesh, India \\ ${ }^{1}$ sateeshkumargudla@gmail.com \\ 2 muvvala_sai@yahoo.co.in \\ * Dept. of ECE, Andhra University, Visakhapatnam, Andhra Pradesh, India \\ 3. gsasi.gps@gmail.com
}

\begin{abstract}
The GPS receiver position can be estimated either by using code or carrier phase pseudorange measurements. The navigation solution obtained using the carrier phase based measurements is more accurate than the code based pseudoranges. It is mainly due to the carrier phase wavelength of the GPS satellite transmitted on L-band signal is very small (i.e. $19 \mathrm{~cm}$ for L1) compared to the code wavelength (i.e. $293 \mathrm{~m}$ for $\mathrm{C} / \mathrm{A}$ code). The receiver cannot accurately determine the integer number of wavelengths. Therefore, the carrier phase measurements observed at the receiver have some ambiguity in its estimation called integer ambiguity (integer number of carrier phase cycles). The key to precise carrier phase based positioning is to resolve these integer ambiguities which is extremely challenging when more noise or jamming is present. In this paper, a precise navigation solution algorithm based on integer ambiguity free carrier phase measurements is presented. This algorithm uses ambiguity free carrier phase measurements as well as least squares method.
\end{abstract}

Keyword- GPS, Pseudorange, Carrier phase measurements

\section{INTRODUCTION}

The Global Positioning System (GPS) is a reliable, all weather satellite based radio navigation system that provides accurate three dimensional (3D) navigation solution i.e. position, velocity and timing information up to $10^{-6}$ seconds anywhere on or above the earth's surface. GPS is the only system today able to provide the exact position on the earth at anytime, anywhere, and in all weather conditions [1]. GPS has provided worldwide location finding capability and has helped in developing many location aware applications. The GPS system consists of a nominal 24 satellites, distributed evenly in 6 orbital planes around the globe at an altitude of about $20,200 \mathrm{~km}$. Each satellite transmits two frequencies of signals $\left(\mathrm{L}_{1}=1575.42 \mathrm{MHz}\right.$ and $\left.\mathrm{L}_{2}=1227.60 \mathrm{MHz}\right)$. The GPS receiver position can be estimated either by using code or carrier phase pseudorange measurements. The navigation solution obtained using the carrier phase based measurements is more accurate than the code based pseudoranges. It is mainly due to the carrier phase wavelength of the GPS satellite transmitted on L-band signal is very small (i.e. $19 \mathrm{~cm}$ for L1) compared to the code wavelength (i.e. $293 \mathrm{~m}$ for C/A code). In case of carrier phase measurements, the distance between satellite and receiver is estimated by determining the number of wavelengths underlying the GPS signal. The receiver can measure the fractional part of a cycle with an accuracy of $1 / 100$. That is, the pseudorange measurement is of the order of $1.9 \mathrm{~mm}(19 \mathrm{~cm} / 100)$ and the corresponding position accuracies will be in centimeter level. However, the receiver cannot accurately determine the integer number of wavelengths. Therefore, the carrier phase measurements observed at the receiver have some ambiguity in its estimation called integer ambiguity (integer number of carrier phase cycles). The key to precise carrier phase based positioning is to resolve these integer ambiguities which is extremely challenging when more noise or jamming is present. In this paper, a precise navigation solution algorithm based on integer ambiguity free carrier phase measurements is presented. This algorithm uses ambiguity free carrier phase measurements as well as least squares method.

\section{Carrier Phase Measurement}

The computation of navigation solution involves estimation of i) satellite positions due to broadcast ephemerides, and ii) pseudorange measurements obtained due to code or carrier phase measurements. The receiver position can be estimated either by using code or carrier phase pseudorange measurements. The navigation solution obtained using the fact that the carrier phase measurements is more accurate than the code based pseudoranges. A measurement much more precise than that of code phase measurements is the phase of the carrier received from a satellite. The carrier phase measurement is the difference between the phases of the receiver-generated carrier signal and the carrier received from a satellite at the instant of measurement. The phase of the received signal at any instant can be related to the phase at the time of transmission in terms of the transit time of the signal. Though these carrier phase measurements are precise, they are ambiguous. Using 
carrier phase, distance between a satellite and the receiver would be an unknown number of cycles plus the measured fractional cycle. The measurement however contains no information regarding the whole cycles referred to as the integer ambiguity.

Even with the advances in the code measurement technologies, a far more precise measurement than the pseudorange is the carrier phase measurement. The carrier phase measurement is the difference in phase between the transmitted carrier wave from the satellite and the receiver oscillator signal at a specified epoch [2]. The range is simply the sum of the total number of full carrier cycles between the receiver and the satellite, multiplied by the carrier wavelength. The ranges determined with the carriers are far more accurate than those obtained with the codes. This is due to the fact that the wavelength (or resolution) of the carrier phase, $19 \mathrm{~cm}$ in the case of L1 frequency, is much smaller than those of codes [6]. The receiver, when switched on cannot determine the total number of the complete cycles between the satellite and receiver. It can only measure a fraction of a cycle very accurately, while the initial number of complete cycles remains unknown, or ambiguous. This is commonly known as the initial cycle ambiguity, or the ambiguity [4].

Figure 1 gives an illustration of unknown number of integer cycles that are elapsed before the receiver locks the phase of the incoming GPS signal. Precise GPS positioning relies on tracking the carrier-phase. The fractional part of carrier-phase can be measured directly using a standard phase-locked loop, but the integer part is ambiguous and the ambiguity must be resolved based on sequential carrier phase measurements to ensure the required positioning precision. In general, centimeter-level GPS positioning accuracy requires precise tracking of the carrier phase that consists of two parts: a directly measured fractional part (with measurement error at millimeter level) and an unknown integer part, also called the integer ambiguity. The key to precise carrier phase based positioning is to resolve the integer ambiguity.

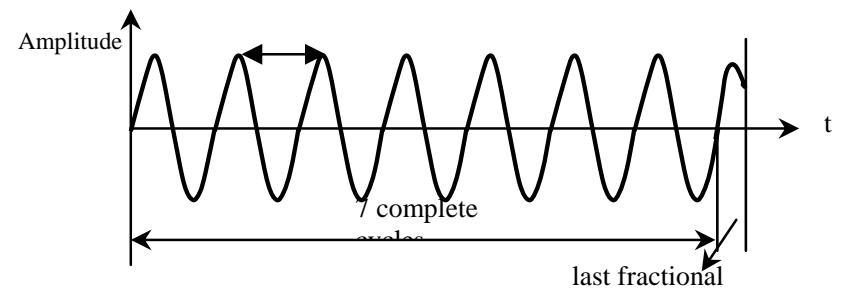

Figure 1 Illustration of the integer ambiguities

\section{III.New Navigation Solution Based On InTeger Ambiguity Free CarRier Phase Measurements}

The proposed new navigation solution algorithm is a three-step procedure developed for precise positioning. First step is to compute the float solution. Any suitable method can be used here, for example ordinary leastsquares, or in recursion a Kalman filter approach. Second step is to determine the integer values for the ambiguities. The developed new navigation solution algorithm takes the estimated float solution $\hat{N}$, together with its variance-covariance matrix $\Sigma_{\hat{N}}$ as input, and delivers fixed integer values. Finally, again using existing algorithm, the eventual fixed solution can be obtained. In this paper, the least squares iterative method is used for float solution [5].

\section{Algorithm for fixing the integer values of the ambiguities}

The main feature of this method is the decorrelation of the ambiguities via the Z-transformation and this ambiguity fixing process follows two steps.

Step 1: Disregard the constraint on the ambiguities and obtain a solution $[\hat{x} \hat{N}]$, which is obtained in the above discussion. Now find the covariance matrix

$$
\operatorname{cov}\left[\begin{array}{l}
\hat{x} \\
\hat{N}
\end{array}\right]=\left[\begin{array}{cc}
\sum_{\hat{X}} & \sum_{\hat{x}, \hat{N}} \\
\sum_{\hat{x}, \hat{N}} & \sum_{\hat{N}}
\end{array}\right]
$$

where, $\hat{x}, \hat{N}$ are the float solutions for linearised observation equation and integer ambiguities $\sum_{\hat{x}}$ and $\sum_{\hat{N}}$ are the corresponding covariance matrices

$\sum_{\hat{x}, \hat{N}}$ gives the cross correlations between $\sum_{\hat{x}}$ and $\sum_{\hat{N}}$.

Step 2: Find the integer vector $\mathrm{N}$ which minimizes the function

$$
(N-\hat{N})^{T} \Gamma^{-1}(N-\hat{N})
$$


where $\hat{N}$ is the float solution from step 1 and $\Gamma$ is inverse of covariance matrix $\Gamma=\Sigma_{\hat{N}}^{-1}$. Step 2 is very important in lambda method. The measure of distance (or,'nearness') of an integer vector $\mathrm{N}$ is given by above equation. The contour of points with a constant value of the function is an ellipse in two dimensions and an ellipsoid in higher dimensions, centered at $\hat{N}$. The search space is delimited by selecting the size of the ellipsoid to be searched via a parameter value $\mathrm{c}>0$. The inequality

$$
(N-\hat{N})^{T} \Gamma^{-1}(N-\hat{N}) \leq c
$$

Eq.(3) defines the integer vectors $\mathrm{N}$. The search space consists of grid points inside an ellipsoid. Clearly, this search space must be large enough to contain the right answer and small enough to be searched quickly[3]. If ' $\Gamma$ ' is diagonal the solution for $\mathrm{N}$ can be found by rounding each component of $\hat{N}$ to its nearest integer. A diagonal $\Gamma$ would mean that the integer ambiguity estimates in float solution are all uncorrelated. This simple approach, however, does not work in realistic situations where the matrix, $\Gamma$ is typically not diagonal. Therefore, use step 2 to introduce a change of variables so that resultant correlation matrix is diagonal. $\Gamma$ is a positive semi-definite matrix and it would appear that diagonalizing it would not be a problem. Use the matrix of its eigen vectors to transform the variables. Actually this approach will not work here, because the transformation will not preserve the integer nature of ambiguities. To restrict the transformations to those that take integers into integers. Actually the inverse transformation $\mathrm{Z}$ must also do the same so that, find the solution of the original problem. The required transformation $\mathrm{Z}$ must satisfy the following conditions.

\section{$\mathrm{Z}$ must have integer entries. \\ $\mathrm{Z}$ must be invertible, and}

$\mathrm{Z}^{-1}$ must have integer entries.

These conditions ensure that there is a one-to-one relationship between the integers in the original and transformed spaces. Consider a hypothetical transformation $\mathrm{Z}$ in this restricted class of transformations with $\Gamma$.

$$
\text { Consider } \mathrm{M}=\mathrm{ZN} \text { and } \hat{M}=\mathrm{ZN}
$$

The function in (Eq.4) in the transformation space is

$$
(M-\hat{M})^{T}\left(Z^{-T} \Gamma^{-1} Z^{-1}\right)(M-\hat{M})
$$

Since $\left(Z^{-T} \Gamma^{-1} Z^{-1}\right)$ is diagonal, now find the solution for $\mathrm{M}$ right away by the rounding off each element of $\hat{M}$ and obtain the integer vector ' $\mathrm{N}$ ' by inverse Z-transformation to the $\mathrm{M}$.

$$
\mathrm{N}=\mathrm{Z}^{-1} \mathrm{M}
$$

Step3: obtain fixed solution, $\mathrm{x}$ after fixing integer ambiguities to $\mathrm{N}$ found in step2.

$$
x=\left(A^{T} A\right)^{-1} A^{T}(y-B N)
$$

where,

$A=$ observation matrix of linearised observation equation

$\mathrm{B}=$ identity matrix of linearised observation equation.

\section{IV.RESULTS AND DisCUSSION}

The implementation of the proposed navigation solution algorithm involves computation of satellite positions due to broadcast ephemeris and pseudorange measurements obtained due to code and carrier phase measurements. The accuracy of the obtained receiver position from the proposed navigation solution algorithm is validated with the real time dual frequency receiver data obtained from Andhra University Engineering College, Visakhapatnam (Lat/Lon:17.73 $\mathrm{N} / 83.319^{0} \mathrm{E}$ ), and IISc Bangalore (Lat $/$ Lon: $13.02^{0} \mathrm{~N} / 77.57^{0} \mathrm{E}$ ). Figure 2 shows the position error ( $\mathrm{x}-, \mathrm{y}-, \mathrm{z}-)$ in meters as a function of time with reference to the true position of the GPS receiver from their respective mean values for different epochs (time intervals) using least squares method. The maximum values of $\mathrm{x}-, \mathrm{y}-$, $\mathrm{z}$ - position errors are $10.87 \mathrm{~m}, 22.94 \mathrm{~m}$ and $8.342 \mathrm{~m}$ respectively. The minimum values of $\mathrm{x}-\mathrm{y}$-, $\mathrm{z}$ - position errors are $-30.43 \mathrm{~m},-13.34 \mathrm{~m}$, and $-18.19 \mathrm{~m}$ respectively. 

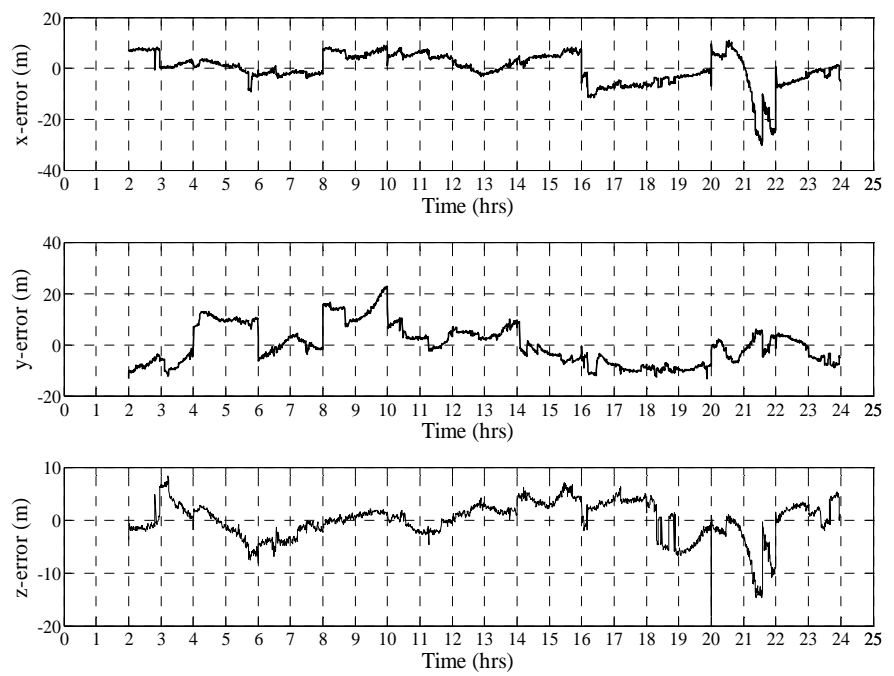

Figure 2 Position error in (x-, y-, z-) coordinate system vs. Time (in hours) of the day (21 ${ }^{\text {st }}$ December 2011) due to least squares method
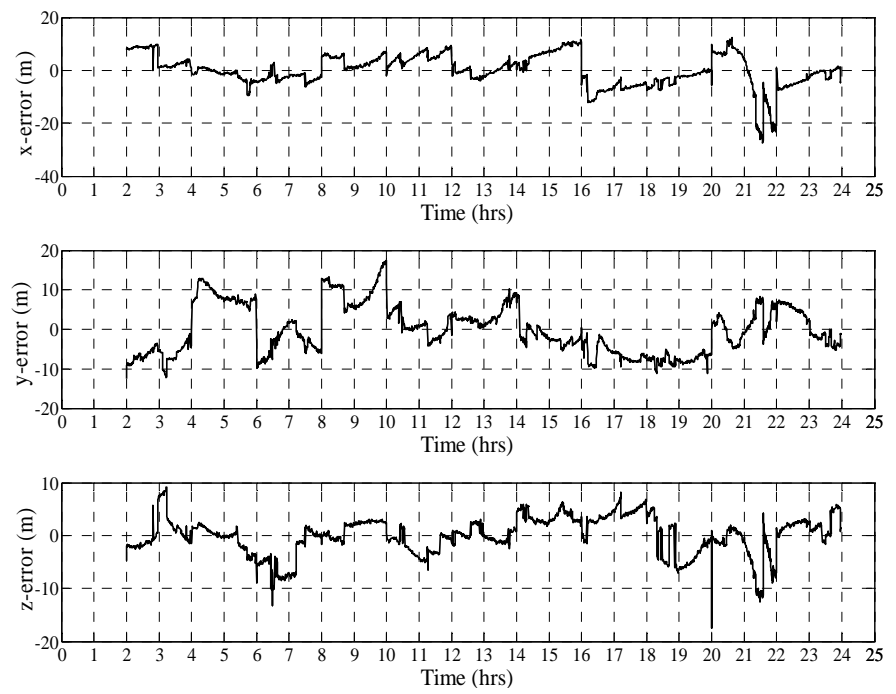

Figure 3 Position error in (x-, y-, z-) coordinate system vs. Time (in hours) of the day (21 $1^{\text {st }}$ December 2011) due to proposed navigation solution algorithm

Figure 3 shows the variation of position error ( $\left.x-, y-, z^{-}\right)$in meters as a function of time with reference to the true position of the GPS receiver from their respective mean values for different epochs (time intervals) using proposed navigation solution algorithm. The maximum values of $\mathrm{x}-$, $\mathrm{y}-, \mathrm{z}$ - position errors are $12.35 \mathrm{~m}, 17.59 \mathrm{~m}$ and $9.144 \mathrm{~m}$ respectively. The minimum values of $\mathrm{x}-, \mathrm{y}-, \mathrm{z}$ - position errors are $-27.38 \mathrm{~m}, 12.54 \mathrm{~m}$ and $-17.51 \mathrm{~m}$ respectively. Table 1 Comparison between least squares method and the proposed new navigation solution algorithm.

Table 1 Comparison between least squares method and the proposed new navigation solution algorithm

\begin{tabular}{|c|c|c|c|c|c|c|c|c|}
\hline \multirow{2}{*}{$\begin{array}{c}\text { Receiver } \\
\text { position } \\
\text { error }\end{array}$} & \multicolumn{4}{|c|}{ Least squares method } & \multicolumn{3}{c|}{ Proposed navigation algorithm } \\
\cline { 2 - 10 } & $\begin{array}{c}\text { Maxim } \\
\text { um (m) }\end{array}$ & $\begin{array}{c}\text { Minim } \\
\mathbf{u m}(\mathbf{m})\end{array}$ & $\begin{array}{c}\text { Standard } \\
\text { deviation } \\
\mathbf{( m )}\end{array}$ & $\begin{array}{c}\text { Mean } \\
\mathbf{( m )}\end{array}$ & $\begin{array}{c}\text { Maxim } \\
\mathbf{u m} \text { (m) }\end{array}$ & $\begin{array}{c}\text { Minim } \\
\mathbf{u m} \\
\mathbf{( m )}\end{array}$ & $\begin{array}{c}\text { Standard } \\
\text { deviation } \\
\text { (m) }\end{array}$ & $\begin{array}{c}\text { Mean } \\
\text { (m) }\end{array}$ \\
\hline x- position error & 10.87 & -30.43 & 6.245 & -32.90 & 12.35 & -27.38 & 6.04 & -33.60 \\
\hline y- position error & 22.94 & -13.34 & 7.66 & 32.98 & 17.59 & -12.54 & 6.237 & 28.33 \\
\hline z- position error & 8.342 & -18.19 & 3.543 & 4.64 & 9.144 & -17.51 & 3.571 & 3.93 \\
\hline $\begin{array}{c}\text { RMS position } \\
\text { error }\end{array}$ & 27.36 & -14.76 & 6.294 & 47.56 & 26.75 & -14.51 & 6.081 & 44.70 \\
\hline
\end{tabular}




\section{CONCLUSION}

In general, centimeter-level GPS positioning accuracy is necessary in all precise navigation solution applications such as aircraft navigations, missile guidance and surveying. In this paper, a new precise navigation solution algorithm based on integer ambiguity free carrier phase measurement is presented. In this method, first the integer ambiguities are estimated using least squares method. These estimated ambiguities are resolved (or fixed to integer values) by decorrelation of the ambiguities using Z-transformation. These fixed integer values are added to the carrier phase measurements and then the positioning is done using least squares iterative algorithm. It is observed that the receiver position obtained from the ambiguity free carrier phase measurements are more accurate than the position obtained from code range measurements as well as from the carrier phase measurements without ambiguity correction.

\section{REFERENCES}

[1] Rao G. S., “Global Navigation Satellite Systems with Essentials of Satellite communications”, McGraw Hill, New Delhi, India, May 2010.

[2] Azimi-Sadjadi B, Krishna Prasad P.S., “Integer ambiguity resolution in GPS using particle filtering”, Proc. Am Control Conf 5, pp 3761-3766, 2001.

[3] Sasibhushana Rao G., "GPS satellite and receiver instrumental biases estimation using least squares method for accurate ionosphere modelling”, Journal of Earth System Sciences, Vol. 116, No. 5, October 2007, pp. 407-411.

[4] Hassibi A. and Boyd S., "Integer parameter estimation in linear models with applications to GPS”, IEEE Trans Signal Proces. 46, pp 2938-2952, 1998

[5] Xu G., "GPS Theory, Algorithms and Applications”, Springer-Verlag, New York, USA, 2003.

[6] Rabbany A. El, “Global Positioning System”, Artech House Publishers 2002.

\section{AUTHOR PROFILE}

Dr. G. Sateesh Kumar completed his Ph.D from Andhra University in the Dept. of Electronics and Communication Engineering. He has 12experience years in teaching and research. He has published more than 15 National/ International Journals/ Conferences. He is a life member of ISTE. Presently he is working as Professor in Electronics \& Communication Engineering Department. AITAM, Tekkali.

Dr. M N V S S Kumar completed his Ph.D from Andhra University in the Dept. of Electronics and Communication Engineering. He has 6experience years in teaching and research. He has published more than 90 National/ International Journals/ Conferences. Presently he is working as Associate Professor in Electronics \& Communication Engineering Department. AITAM, Tekkali.

Prof. G Sasi Bhushana Rao has 28 years of experience in R\&D, Industrial and Teaching. He was involved in the development of GAGAN system which is jointly developed by the ISRO and AAI. He has published more than 400 research papers in various reputed International/ National Journal/Conferences. He is a Senior Member of IEEE, FIETE, IEEE Com Society, IGU and Member of International Global Navigation Satellite System (IGNSS), Australia. His area of research includes GPS/INS Signal Processing, RADAR, SONAR and Acoustic signal modeling. Presently he is working as Professor in Electronics \& Communication Engineering Department, Andhra University Engineering College, Visakhapatnam. 